\title{
POSTER
}

\section{À propos d'un cas d'allogreffe osseuse maxillaire pré implantaire assistée par ordinateur}

\author{
Feugueur $\mathrm{G}^{\star}$, Keribin $\mathrm{P}^{\star}$, Grolleau $\mathrm{P}^{\star}$, Moizan $\mathrm{H}^{\star}$ \\ *Service odontologie Hôpital Saint Julien CHU Rouen
}

L'augmentation de volume osseux crestal pré implantaire peut constituer aujourd'hui un réel défi chez certains patients dès lors que l'on souhaite obtenir une reconstitution implanto-prothétique stable, esthétique et pérenne. Différentes modalités d'augmentation du volume osseux maxillaire existent selon l'origine du greffon. Ainsi l'autogreffe osseuse, considéré encore comme le gold standard requiert un prélèvement peropératoire intra ou extra-oral. Les greffes osseuses allogéniques ont l'avantage de permettre de s'exonérer d'un prélèvement sur un site donneur et ainsi de diminuer la morbidité de cette chirurgie avec un taux de succès équivalent à l'autogreffe (Esposito et al., 2009 cochrane). Les techniques de reconstruction osseuses faisant appel aux substituts osseux avec membrane armée en titane montrent leurs limites en termes de hauteur de volume régénéré à $7 \mathrm{~mm}$ environ (Simion et al., 2001 clin oral impl res). La demande croissante de réhabilitations implantaires nous conduit à prendre en charge des patients de plus en plus âgés avec de ce fait très souvent des co-morbidités associées. Le concept d'élaboration d'un greffon osseux sur mesure à partir d'une imagerie tomodensitométrique est de ce fait séduisant pour le chirurgien oral en raison du gain de temps chirurgical en évitant le prélèvement sur un site donneur ainsi que la phase de découpe et de façonnage du greffon, tout en améliorant de façon significative la congruence de la surface du greffon en regard du site receveur et en facilitant son ostéosynthèse réduisant ainsi les risques de micro-mobilité et donc d'échec ( Antoun et al, 2013 aos).

C'est à partir de cette innovation proposée par une société prestataire fournissant le greffon osseux sur mesure que nous vous présentons le cas clinique de reconstitution pré-implantaire maxillaire chez une patiente de 81 ans. Toutes les étapes depuis la consultation initiale, le bilan scannographique en passant par le choix du type du greffon (spongieux ou cortico-spongieux), la modélisation et conception 3D du greffon, et la chirurgie reconstructrice pré-implantaire sous anesthésie locale seront exposées. La réduction du temps chirurgical à moins de 45 minutes, les suites opératoires diminuées et le confort de notre patiente nous autorisent à penser que dans l'avenir ces techniques vont se développer du fait de nombreux avantages tant pour le chirurgien que le patient. 